OPEN ACCESS

Edited by:

Vincent Vander Poorten,

KU Leuven, Belgium

Reviewed by:

Ashok R. Shaha,

Memorial Sloan Kettering Cancer

Center, United States

Shilpi Sharma,

Tata Memorial Hospital, India

${ }^{*}$ Correspondence:

Rong Zheng

zrong99@163.com

Specialty section:

This article was submitted to

Head and Neck Cancer,

a section of the journal

Frontiers in Oncology

Received: 03 March 2019 Accepted: 07 June 2019

Published: 28 June 2019

Citation:

Yang J, Zheng R, Liang M, Jia Y, Lin L,

Geng J, Chen S and Li Y-X (2019)

Association of the Cumulative Dose of

Radioactive lodine Therapy With

Overall Survival in Patients With

Differentiated Thyroid Cancer and

Pulmonary Metastases.

Front. Oncol. 9:558.

doi: 10.3389/fonc.2019.00558

\section{Association of the Cumulative Dose of Radioactive lodine Therapy With Overall Survival in Patients With Differentiated Thyroid Cancer and Pulmonary Metastases}

\author{
Jing Yang ${ }^{1}$, Rong Zheng ${ }^{1 *}$, Meng Liang ${ }^{1}$, Yingying Jia ${ }^{1}$, Lin Lin ${ }^{1}$, Jianhua Geng ${ }^{1}$, \\ Shengzu Chen ${ }^{1}$ and Ye-Xiong $L i^{2}$ \\ ${ }^{1}$ Department of Nuclear Medicine, National Cancer Center, National Clinical Research Center for Cancer, Cancer Hospital, \\ Chinese Academy of Medical Sciences and Peking Union Medical College, Beijing, China, ${ }^{2}$ Department of Radiation \\ Oncology, National Cancer Center, National Clinical Research Center for Cancer, Cancer Hospital, Chinese Academy of \\ Medical Sciences and Peking Union Medical College, Beijing, China
}

Purpose: The optimal cumulative dose of radioactive iodine therapy (RAIT) for patients with differentiated thyroid cancer (DTC) and pulmonary metastases (PM) is not known, therefore we evaluated the association between the cumulative dose of RAIT and overall survival (OS).

Methods: A total of 202 patients with DTC and PM who underwent thyroidectomy and RAIT were analyzed in this study. The median cumulative dose of RAIT was $530 \mathrm{mCi}$. OS was compared with an age- and sex-matched general population from China to assess relative survival. Multivariable proportional hazards model smoothing by penalized spline was applied to identify independent predictors and examine the adjusted non-linear association of cumulative dose of RAIT and patient age with mortality.

Results: The observed survival and relative survival at 10 years was 54.96 and $60.81 \%$, respectively, with the standardized mortality ratio being 5.34. The cumulative dose of RAIT was associated with mortality in a dose-dependent fashion without an apparent cutoff point after adjustment of other variables. A linear but moderate association was found in the dose of 300 to $1,000 \mathrm{mCi}$. Cumulative dose of RAIT, patient age, diameter of pulmonary metastases, and extrapulmonary metastases were identified as independent predictors for OS. The increasing patient age was associated with mortality in a non-linear pattern, with the optimal threshold being 40 years. With advancing age, the risk of death increases rapidly in patients aged 40 years and younger, but slowly in patients over 40 years.

Conclusions: RAIT should be assigned to RAl-avid patients until disease has been controlled or RAIT becomes refractory after consideration of the potential long-term side-effects. Patient age was associated with OS in a non-linear pattern, with a threshold at 40 years. Consideration of age as a binary variable could elucidate a more accurate prognosis in such patients.

Keywords: differentiated thyroid cancer, pulmonary metastases, radioiodine therapy, cumulative dose, patient age 


\section{INTRODUCTION}

The incidence of thyroid cancer (TC) has increased persistently worldwide in recent years (1). In China, annual newly diagnosed cases of TC has increased from 54,175 in 2010 to 201,000 in $2015(2,3)$, of which differentiated thyroid cancer (DTC) accounts for $>90 \%$. Five-year survival for TC is higher than that for all other cancer sites, and reached $84.3 \%$ in 2012 2015 in China (4). However, patients with distant metastases carry an increased risk of death from TC (5). The lungs are the most common organ of distant metastases, accounting for $\sim 70 \%$ of all cases of distant metastases (6). DTC patients with pulmonary metastases $(\mathrm{PM})$ have a relatively poor prognosis, with median survival $<10$ years ( 7 ).

For patients with DTC and PM, consensus guidelines recommend that radioactive iodine therapy (RAIT) should be introduced after a total or near-total thyroidectomy to reduce the risk of disease recurrence or mortality $(8,9)$. Also, RAIT should be first-line therapy for DTC patients as long as the inoperable PM are RAI-avid (10). RAIT courses in patients with DTC and PM are generally based on responses to RAIT. The following course of RAIT would be delivered to patients who concentrate RAI and respond clinically within PM $(6,11)$. With regard to the cumulative RAI dose, limits at 600 or 1,000 mCi have been recommended in several studies, but they were empiric settings without adjustment for other variables $(12,13)$. Studies have deemed that patients with RAI-avid PM could obtain survival benefit from RAIT (14). However, whether increasing amount of RAIT translates into long-term survival benefit for such patients is not known (15).

In patients with DTC and PM, patient age is the most important prognostic factor (16). Moreover, it has been reported that age has an association with the response to RAIT (16, 17).Therefore, taking age into account while evaluating the effect of the cumulative dose of RAIT on overall survival (OS) is crucial. Most studies have incorporated age into binary variables with a distinct cutoff point for DTC patients (18). In 2018, the 8th edition of the Staging System for Primary Tumors set by the American Joint Committee on Cancer adjusted the age cutoff point from 45 to 55 years, raising concerns among pathologists and clinicians (19-21). Recent studies using age as a continuous variable rather than a dichotomic factor found better concordance with survival and death from TC $(22,23)$. For patients with DTC and PM, studies have demonstrated that an age of $\geq 45$ years carries a worse prognosis than in patients aged $<45$ years $(24,25)$. However, no study has examined the dose-dependent effect of age among patients with DTC and PM.

In this patient cohort with long-term follow-up, we aim to ascertain if there is a dose-dependent effect for RAIT and survival benefits in patients with DTC and PM. Furthermore, we try to examine if there is an optimal dose of RAIT and an age cutoff for survival in patients with DTC and PM.

\section{MATERIALS AND METHODS}

\section{Patient Population}

We identified cases with a diagnosis of DTC and PM in the National Cancer Center of China, and restricted patient age at diagnosis of PM to older than 18 years. All medical records of DTC patients who had received RAIT in the Department of the Nuclear Medicine of National Cancer Center during this period were reviewed systematically. For RAI-avid patients, PM diagnosis was primarily based on an increased serum level of thyroglobulin $(\mathrm{Tg})$ and positive results in therapeutic iodine 131 (I-131) whole-body scan (WBS), taking pulmonary nodules on radiography or computed tomography (CT) of the chest into consideration. "WBS-positive" was defined by status of I131 uptake in the lungs higher than the normal basal level (excluding physiologic uptake and contamination from the body surface). With regards to non-RAI-avid patients whose pulmonary nodules were WBS-negative throughout the entire treatment, PM were confirmed by radiography or CT of the chest and increased serum level of $\mathrm{Tg}$ during follow-up.

\section{Treatment}

All patients underwent thyroidectomy. When necessary, more than one procedure would be performed on patients to ensure total or near-total thyroidectomy. Central lymph node compartment (level VI lymph nodes) dissection was performed routinely except for $\mathrm{cN} 0$ patients with micropapillary carcinoma or well-differentiated follicular carcinoma. Therapeutic lymph node neck dissection would be performed if a suspicious lymph node was detected by pre-operative imaging or physical examination or intraoperative exploration, while prophylactic lymph node dissection was not actively performed. In order to ensure the concentration of radioactive iodine in pulmonary metastases, all patients received surgical treatment before RAI therapy and reoperation would be delivered to the patients who confirmed with cervical lymph node metastasis during RAI therapy. After thyroidectomy, conventional measurements including free tri-iodothyronine, iodothyronine, free thyroxine, thyroxine, thyroid-stimulating hormone (TSH), $\mathrm{Tg}$, ultrasonography of the neck, and CT/radiography of the chest were done before the first course of RAIT. Then, patients were instructed to follow a low-iodine diet for $\geq 2$ weeks. During the 3-4 weeks before the initiation of RAIT, patients were not allowed to take any iodine or iodine-containing substance in order to guarantee the withdrawal of thyroid hormones to achieve an adequate hypothyroid state (serum level of TSH $>30.00 \mathrm{uIU} / \mathrm{mL}$ ).

The radioactive iodine therapy was carried out using fixeddose method taking into account the physical status of patients. All patients were administered thyroid-hormone therapy after RAIT for $24-48 \mathrm{~h} .{ }^{131}$ I-WBS was carried out 3-8 days after oral RAIT to observe RAI absorption in PM, the results of which, combined with a change in serum levels of $\mathrm{Tg}$ and tumor sizes on anatomic imaging, were considered to be prognostic indicators for the assessment of PM response to RAIT. After a course of RAIT, patients with smaller or less metastatic pulmonary nodules in imaging examinations, reduced concentration of iodine within PM in I-131-WBS, and reduced Tg levels (compared with inhibition or stimulation of TSH) than that before this administration were thought to be effective. Clinicians made subsequent treatment plans based on the therapeutic response of last course of RAIT. The RAIT would stop once a patient was 
diagnosed as non-RAI-avid PM, and generally received a referral for radiotherapy or chemotherapy.

\section{Endpoint and Statistical Analyses}

All patients were observed at follow-up even if they were transferred to other hospitals or departments. Patients who completed the entire treatment in our hospital were followed up annually for the first 5 years and then every 2 or 3 years. Patients transferred to other hospitals were followed up by correspondence or telephone contact. Statistical analyses were undertaken on follow-up data collected up to September 2017 or at the time of death from any cause. The primary endpoint was OS (defined from the time of the initial diagnosis of PM to the time of death from any cause or to the final followup assessment).

The overall survival was compared with data from an ageand sex-matched population to evaluate the net survival (relative survival) of this cohort of patients with DTC and PM. The Kaplan-Meier method was used to estimate observed survival. Expected OS accounting for age and sex was generated in the $\mathrm{R}$ program (Vienna, Austria) using the general population of China as a reference group. Observed survival vs. expected OS was plotted using a conditional approach and expressed as a standardized mortality ratio (SMR) of observed deaths to expected deaths. Then, relative survival was estimated by the Ederer II method to account for competing deaths from other causes. Cox proportional hazards regression with a stepwise procedure was used to identify the independent predictors for OS. The penalized spline (P-spline) fitting the Cox model allowed a non-linear relationship of cumulative dose of RAIT and age with the logarithm (ln hazard ratio [LnHR]) of mortality estimated from the full Cox regression model adjusted for other independent covariates.

\section{RESULTS}

\section{Patient Characteristics}

A total of 202 patients with DTC and PM formed the study cohort. The median age was 50.5 (range, 18-86) years and $57 \%$ were female (Table 1). Most patients had: papillary thyroid carcinoma (73\%); lymph-node involvement (76\%); no extrapulmonary metastases $(81 \%)$; iodine concentration within PM (74\%); not undergone radiotherapy or chemotherapy (79\%). All 202 patients underwent thyroidectomy and 167 patients received central cervical lymph node dissection. Only central lymph node dissection was delivered to 52 patients, while additional lateral lymph node dissection was delivered to 115 patients (43 patients received bilateral lymph node dissection and 72 patients received unilateral lymph node dissection). All patients received at least one course of RAIT after thyroidectomy, with the median cumulative dose of RAIT being 530 (range, 1,000-1,650) $\mathrm{mCi}$. Twenty-seven patients developed local recurrence during follow-up, of which, 22 patients had nodal recurrence, and 5 patients had recurrence in the thyroid bed or tracheal wall. Second primary malignancies were identified in six patients after RAI therapy (four cases of lung cancer, two cases of breast cancer). Two patients were diagnosed as having pulmonary fibrosis. Information about other clinical characteristics of patients are listed in Table $\mathbf{1 .}$

TABLE 1 | Demographic, clinical, and treatment characteristics of patients with differentiated thyroid cancer and pulmonary metastases $(N=202)$.

\begin{tabular}{|c|c|}
\hline Characteristic & No. (\%) \\
\hline \multicolumn{2}{|l|}{ Sex } \\
\hline Male & $87(43)$ \\
\hline Female & $115(57)$ \\
\hline \multicolumn{2}{|l|}{ Age } \\
\hline Range & $18-86$ \\
\hline Median (IQR) & $50.5(36,62)$ \\
\hline Mean (SD) & $49.04 \pm 15.74$ \\
\hline \multicolumn{2}{|l|}{ Pathology } \\
\hline PTC & $148(73)$ \\
\hline FTC & $47(23)$ \\
\hline FV PTC & $7(4)$ \\
\hline \multicolumn{2}{|c|}{ Extrathyroidal extension } \\
\hline Yes & $72(36)$ \\
\hline No & $7(3)$ \\
\hline Unknown & $123(61)$ \\
\hline \multicolumn{2}{|c|}{ Lymph-node involvement } \\
\hline Yes & $153(76)$ \\
\hline No & $40(20)$ \\
\hline Unknown & $9(4)$ \\
\hline \multicolumn{2}{|c|}{ Diameter of pulmonary metastases $(\mathrm{cm})$} \\
\hline$\geq 1$ & $65(32)$ \\
\hline$<1$ & $89(44)$ \\
\hline Unknown & $48(24)$ \\
\hline \multicolumn{2}{|c|}{ Extrapulmonary metastases } \\
\hline Yes & $39(19)$ \\
\hline No & $163(81)$ \\
\hline \multicolumn{2}{|c|}{ Extent of surgery (initial procedure) } \\
\hline Total thyroidectomy & $98(49)$ \\
\hline Thyroid lobectomy & $99(49)$ \\
\hline Unknown & $5(2)$ \\
\hline \multicolumn{2}{|c|}{ lodine concentration within pulmonary metastases } \\
\hline Yes & $149(74)$ \\
\hline No & $53(26)$ \\
\hline \multicolumn{2}{|c|}{ Radiotherapy or chemotherapy } \\
\hline Yes & $42(21)$ \\
\hline No & $160(79)$ \\
\hline \multicolumn{2}{|l|}{ Frequency of rait } \\
\hline Range & $1-10$ \\
\hline Median (IQR) & $3.5(2,5)$ \\
\hline Mean (SD) & $4.03 \pm 2.19$ \\
\hline \multicolumn{2}{|c|}{ Cumulative dose (mCi) } \\
\hline Range & $100-1650$ \\
\hline Median (IQR) & $530(300,850)$ \\
\hline Mean (SD) & $598.03 \pm 349.05$ \\
\hline
\end{tabular}

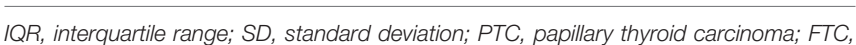
follicular thyroid carcinoma; FV PTC, follicular variant of papillary thyroid carcinoma; RAIT, radioactive iodine therapy. 


\section{Comparison of Survival of the Study Group With That of the General Population}

Median follow-up was 93 (range, 2-258) months. There were 86 deaths during the entire follow-up period. The observed OS was $72.68 \%$ at 5 years and $54.96 \%$ at 10 years (Figure 1). Using the general population of China as a reference, the expected OS was $94.73 \%$ at 5 years and $90.43 \%$ at 10 years, resulting in relative survival of $76.74 \%$ at 5 years and $60.81 \%$ at 10 years (Figure 1). Patients with DTC and PM had significantly decreased survival compared with the age- and sex-matched general population of China, with an overall SMR of 5.34 (95\% confidence interval (CI), 4.32-6.60; $P<0.001)$.

\section{Dose-Dependent Effect of RAIT on OS}

In an unadjusted analysis, estimates of observed OS and relative survival for 202 patients were positively associated with an increasing cumulative dose of RAIT (Table 2). Survival for patients with a cumulative dose $\leq 400 \mathrm{mCi}$ (quartile 1) was lowest, whereas survival for patients with a cumulative dose $>900 \mathrm{mCi}$ (quartile 4) was highest. Survival was relatively similar in patients with a cumulative dose of RAIT in quartile 2 and quartile 3. To quantify the dose-dependent effect, we entered the cumulative dose of $149 \mathrm{RAI}$-avid patients as a continuous variable into the multivariate Cox regression using P-splines to allow for non-linear relationships between the RAIT dose and outcomes. This model confirmed that the risk (adjusted LnHR) of death decreased with an increasing cumulative dose of RAIT, and that

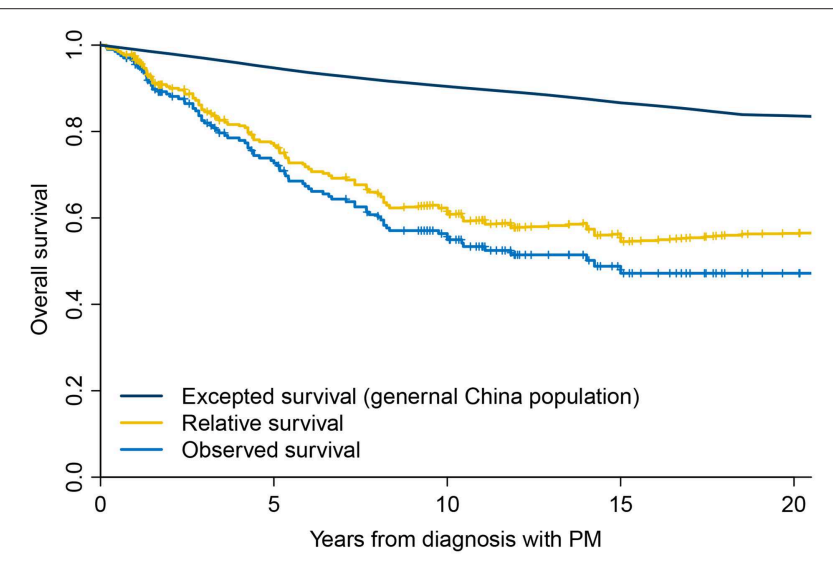

FIGURE 1 | Overall survival of patients with differentiated thyroid cancer and pulmonary metastases compared with the general population of China. the risk was expected to decrease moderately from 300 to 1,000 $\mathrm{mCi}$ (Figure 2). With respect to the 53 non-RAI-avid patients, mortality decreased with an increasing cumulative dose up to 400 $\mathrm{mCi}$ and then plateaued at $500 \mathrm{mCi}$ (Supplementary Figure 1).

Next, we compared the statistical fitness of different multivariate survival models (adjusted by age, sex, pathology type, extrathyroidal extension, lymph-node involvement, extrapulmonary metastases, diameter of pulmonary metastases, radiotherapy, or chemotherapy) with the cumulative dose of RAIT treated as a continuous variable (P-spline or linear) or a binary variable (with cutoff points from 200 to 1,000 $\mathrm{mCi}$ ) or tertile variable (with cutoff points from 200 to 1,000 $\mathrm{mCi}$ ). The model that included the cumulative dose of RAIT as a binary variable with a cutoff point at $750 \mathrm{mCi}$ (Akaike Information Criterion (AIC) $=422.59$ ), and the tertile variable with a cutoff point of 300 and $1,000 \mathrm{mCi}(\mathrm{AIC}=416.43)$ had the lowest AIC estimate compared with other models (Supplementary Figure 2). This finding suggested that treating the cumulative dose of RAIT as a tertile variable provided a better data fit. We further assessed the specific dose-dependent pattern for patients with a cumulative dose between 250 and $1,000 \mathrm{mCi}$, because only five events were observed below or above this range, respectively. As a result, a linear association without an apparent cutoff point between the cumulative dose of RAIT and OS was obtained, thereby reflecting a stable and more accurate dose-dependent relationship (Figure 2).

\section{Independent Predictors of OS}

After stepwise selection of models, the cumulative dose of RAIT, patient age, diameter of pulmonary metastases, and status of extrapulmonary metastases were placed in the best-fitness model. To assess the magnitude of these effects, we included the cumulative dose of RAIT and age as continuous variables for all 202 patients and 149 RAI-avid patients, respectively. The corresponding adjusted HR was 0.86 (95\%CI, 0.82-0.92) and $0.80(0.72-0.88)$ per use of $100 \mathrm{mCi}$ on the cumulative dose of RAIT, and $1.83(1.51-2.21)$ and $1.84(1.48-2.29)$ per 10 year increase in patient age (Figure 3). The risk of death increased for patients with larger diameter of pulmonary metastases and extrapulmonary metastases (Figure 3).

\section{Non-linear Association Between Age and OS}

In an unadjusted analysis, 10 year estimates for observed OS for all 202 patients were inversely associated with increasing age, and the 10 year relative OS confirmed this pattern

TABLE 2 | Ten-year estimates for observed survival and relative survival according to the cumulative dose of radioactive iodine therapy.

\begin{tabular}{|c|c|c|c|c|c|}
\hline Quartile & Cumulative dose (mCi) & $N$ & Events & $\begin{array}{l}\text { Observed survival, \%, } \\
\qquad 95 \% \mathrm{Cl}\end{array}$ & $\begin{array}{c}\text { Relative survival, \%, } \\
95 \% \mathrm{Cl}\end{array}$ \\
\hline 1 & $100.00-400.00$ & 55 & 33 & 37.84 (25.49-56.18) & 41.37 (28.14-60.83) \\
\hline 2 & $400.01-550.00$ & 47 & 20 & 66.27 (49.53-88.67) & 71.45 (53.83-94.84) \\
\hline 3 & 550.01-900.00 & 53 & 21 & 65.59 (51.56-83.43) & 74.39 (58.74-94.22) \\
\hline 4 & $900.01-1650.00$ & 47 & 12 & 80.48 (67.60-95.81) & 86.56 (73.01-98.62) \\
\hline
\end{tabular}




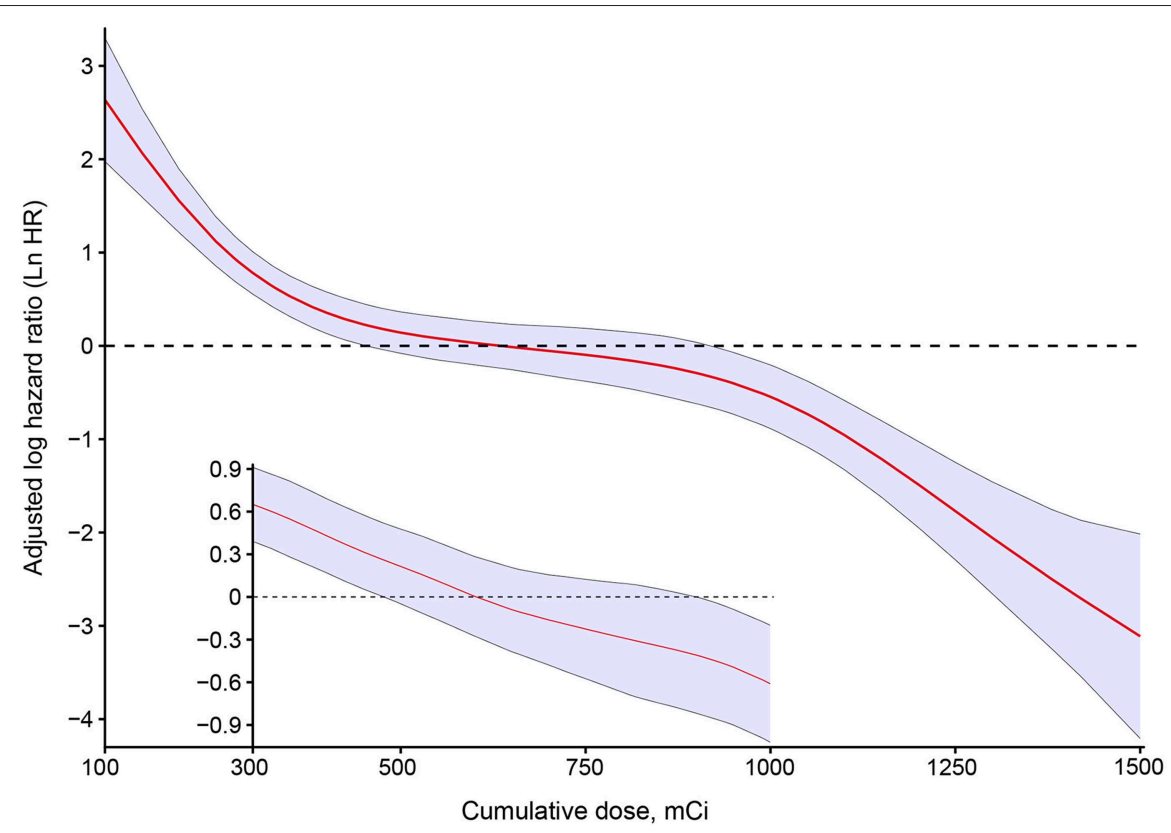

FIGURE 2 | Estimated logarithm hazard ratios with 95\% confidence intervals for the association of the cumulative dose of radioactive iodine therapy and overall survival in patients with differentiated thyroid cancer and pulmonary metastases. Adjusted by age, sex, pathology type, extrathyroidal extension, lymph-node involvement, extrapulmonary metastases, diameter of pulmonary metastases, and radiotherapy or chemotherapy. The main plot indicates the association between the cumulative dose of radioactive iodine therapy and overall survival for all patients with RAl-avid PM. The subset of the plot indicates the association among the cases who received between 300 and $1000 \mathrm{mCi}$.

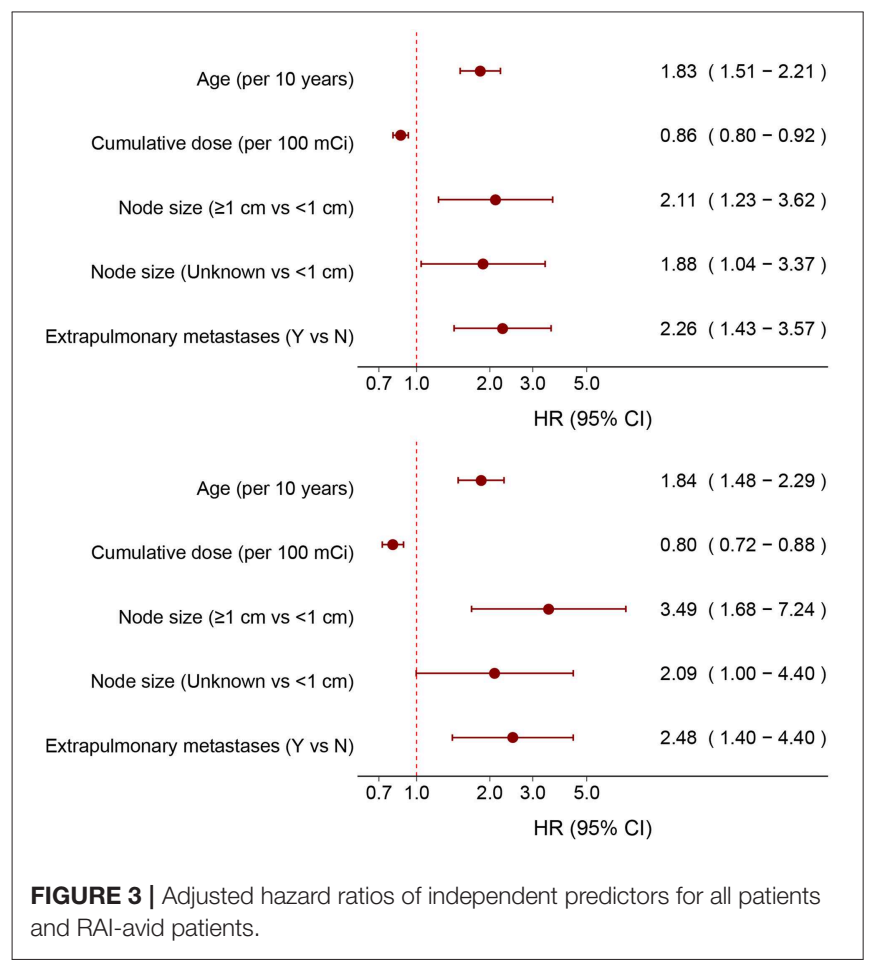

(Table 3). After adjustment for the cumulative dose of RAIT, sex, pathology type, extrathyroidal extension, lymph-node involvement, extrapulmonary metastases, diameter of pulmonary metastases, and radiotherapy or chemotherapy, the P-spline plot demonstrated a significant non-linear association between age and OS in 149 RAI-avid patients, with an apparent cutoff point at $\sim 40$ years (Figure 4). A non-linear association between the predictor and outcome can indicate the existence of a cutoff point in this relationship. Next, we compared the statistical fitness of different age-survival models with age treated as a binary variable (with cutoff points at 18 to 86 years). The model that included age as a binary variable with a cutoff point at 40 years had the lowest AIC (408.74) compared with models that cutoff age at other points (Supplementary Figure 3). This finding suggested that patients aged $>40$ years had an increased risk of death (Supplementary Figure 4).

\section{DISCUSSION}

For patients with DTC and PM, the cumulative dose of RAIT was associated with mortality in a dose-dependent fashion after adjustment for independent prognostic variables. The risk of mortality decreased with an increase in the cumulative dose of RAIT without an apparent cutoff point. A linear but moderate pattern was found in the dose range 300 to $1,000 \mathrm{mCi}$. Patient age, diameter of pulmonary metastases, extrapulmonary metastases, and the cumulative dose of RAIT were independent predictors for OS. Age was associated significantly with mortality in a non-linear pattern after adjustment for clinical and treatment characteristics. The optimal threshold for age was 40 years, 
TABLE 3 | Ten-year estimates for observed survival and relative survival according to age.

\begin{tabular}{|c|c|c|c|c|}
\hline $\begin{array}{l}\text { Age group } \\
\text { (years) }\end{array}$ & $N$ & Events & $\begin{array}{l}\text { Observed survival, \%, } \\
\qquad 95 \% \mathrm{Cl}\end{array}$ & $\begin{array}{c}\text { Relative survival, \%, } \\
95 \% \mathrm{Cl}\end{array}$ \\
\hline $18-29$ & 27 & 0 & 100 & 100 \\
\hline 30-39 & 39 & 3 & 94.20 (86.71-100) & 94.61 (87.19-100) \\
\hline $40-49$ & 30 & 15 & 54.33 (35.24-83.77) & $55.74(36.80-84.43)$ \\
\hline 50-59 & 38 & 19 & 37.17 (20.46-67.52) & 40.34 (22.90-71.08) \\
\hline 60-69 & 51 & 35 & 34.30 (20.29-57.98) & $37.33(20.97-66.45)$ \\
\hline $70-86$ & 17 & 14 & 17.86 (5.35-59.64) & 31.17 (10.93-88.91) \\
\hline
\end{tabular}

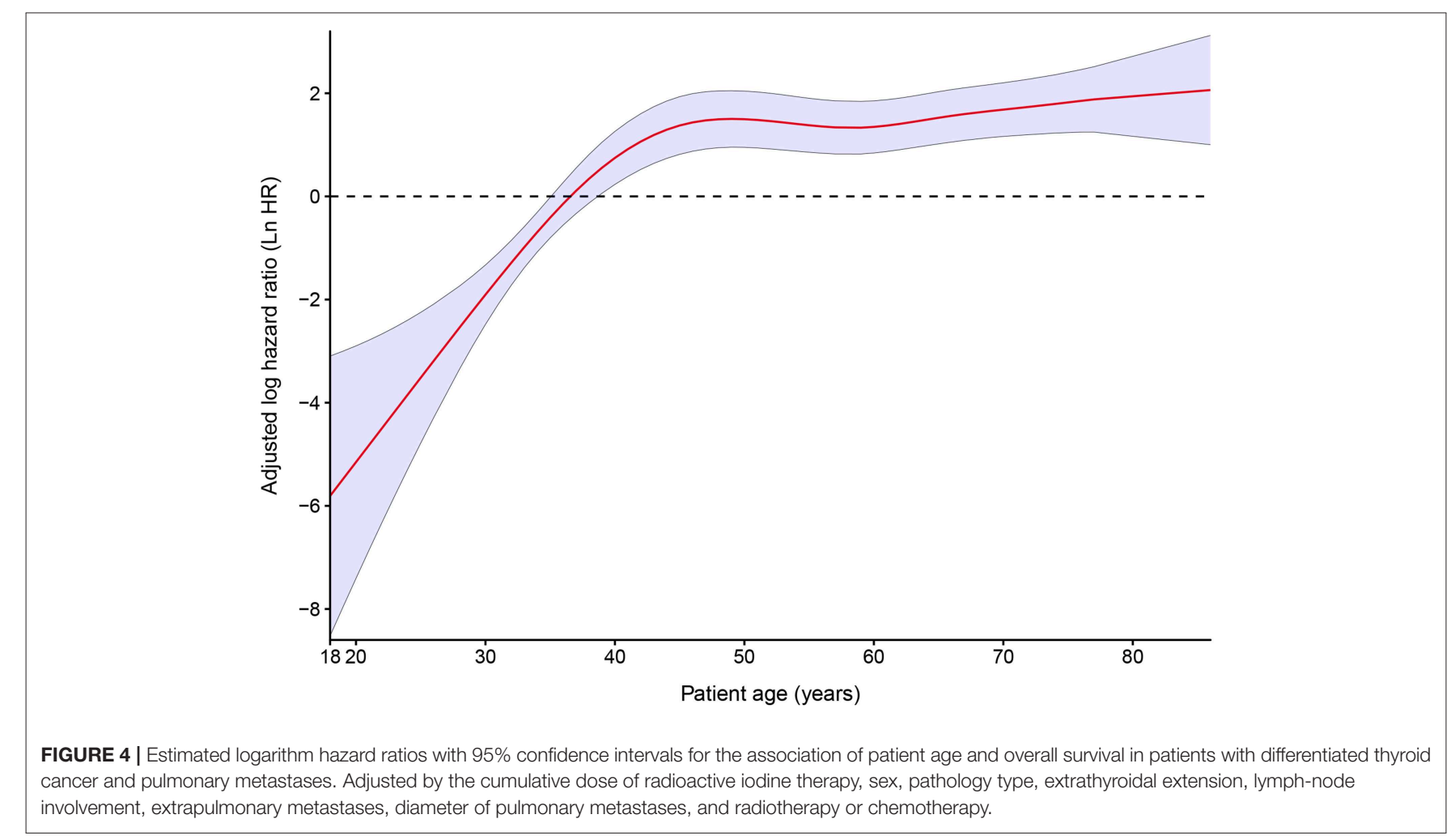

identifying a less rapid increase in the probability of death with advancing age.

The cumulative dose of RAIT was an independent predictor of OS for patients with DTC and RAI-avid PM. A significant association between an increased cumulative dose of RAIT and survival benefit was observed, a finding that is in accordance with other studies $(12,13,26,27)$. Furthermore, we demonstrated that RAIT prolonged survival in a dose-dependent fashion, allowing for linear relationships between the cumulative dose of RAIT and OS. Although the declining trend was moderate from 300 to $1,000 \mathrm{mCi}$, survival outcomes were improved persistently with increased cumulative dose. This pattern may have been a result of a progressive reduction of uptake in the lungs, effective half-life, and the radiation dose delivered to the lungs with successive therapies (28-31). Then, we took patients with a cumulative dose between 300 and $1,000 \mathrm{mCi}$ as a subset group and discovered a linear association without an apparent cutoff point between the cumulative dose of RAIT and OS. These results suggested that an increasing dose had a dose-dependent benefit on survival for patients administered RAIT between 300 and $1,000 \mathrm{mCi}$. The subset analysis may have been more precise because more cases had a dose between 300 and 1,000 mCi. Even though the first two RAIT courses could reduce the mortality risk remarkably, the risk of death for these patients remained high. The effect of the first two courses of RAIT was indicated by Handkiewicz-Junak et al., who found that the recurrence risk was decreased significantly among patients with DTC and distant metastases who received a mean cumulative dose of 300 $\mathrm{mCi}$ of RAIT (32). Patients would not receive further RAIT once PM had been diagnosed as refractory. Thus, patients who received $>1,000 \mathrm{mCi}$ of RAIT were usually RAI-avid consistently during treatment, which resulted in the best prognosis among all patients. Capdevila et al. stated that RAIT can become refractory at a cumulative dose of $600 \mathrm{mCi}$ (33), whereas other scholars had challenged the suggestion that $600 \mathrm{mCi}$ was a threshold to define RAI resistance $(13,34)$. An optimal dose for patients 
with DTC and PM within the range 300 to $1,000 \mathrm{mCi}$ was not found in our study. Our findings suggest that RAIT should be repeated until disease has been controlled or RAIT becomes refractory after consideration of long-term risks. Furthermore, results from 53 non-RAI-avid patients suggested that patients with non-RAI-avid disease usually could not obtain survival benefit from additional RAITs.

In our study, apart from the cumulative dose of RAIT, multivariate analyses suggested that age, diameter of pulmonary metastases, and extrapulmonary metastases status were also independent prognostic factors. A poor prognosis in elder patients with DTC is regarded as common sense by pathologists and clinicians, and recent studies have suggested that age should be treated as a spectrum rather than a specific cutoff point (35). For patients with DTC and PM, the optimal cutoff points for age varied from 40 to 60 years $(17,36-38)$, but no study has taken age as a continuous variable. Our research suggested that the risk of death for patients with DTC and PM increased sharply along with increasing age until 40 years of age, whereas the trend was moderate for patients aged $>40$ years. This non-linear pattern supported studies that had taken 40 years as a cutoff point for prognosis prediction $(7,38)$. The poor prognosis in patients aged $>40$ years could be attributed to a relatively longer disease duration, higher prevalence of advanced disease, poor curative effect of iodine treatment, a decline in the immune system, and a more aggressive variant of TC $(16,39)$. According to our study, consideration of age as a continuous variable or advancement of the cutoff point of age to 55 years for patients with DTC and PM was not applicable, and more evidence is needed to elucidate a more accurate staging system.

Although the lungs are the most common metastatic sites of DTC, population-based data suggest that patients with DTC and distant metastases account for $<1 \%$ of all DTC cases (40). Given the relatively low incidence of patients with DTC and PM, our study provided a relatively large sample size with long follow-up to assess the association between the cumulative dose of RAIT and survival. Our data could be useful for clinicians to decide if they should continue RAI administration in patients with RAI-avid metastatic lung disease despite achieving a cumulative activity $>600 \mathrm{mCi}$. A cutoff point was not found for RAIT, so our study suggests that RAIT should be assigned to RAI-avid patients until disease has been controlled or RAIT becomes refractory after consideration of the long-term side-effects of RAIT. Our data would be helpful to guide RAIT plans for patients with DTC and PM, and might be useful for clinicians to continue the drive toward more individualized management.

Our study had three main limitations. First, this was a retrospective study in a single center in China. A multicenter, international-origin dataset is needed to validate our results. Second, our results were derived from the outcome of allcause deaths. To address this limitation, we used the general population of China as a reference to calculate relative survival from DTC. Third, some patients were RAI-avid initially but non-RAI-avid after several courses of RAITs. We classified these patients into the RAI-avid group according to the initial response, but the underlying variations may have perturbed the dosedependent patterns.

\section{CONCLUSIONS}

In patients with DTC and PM, OS estimates were positively associated with an increasing cumulative dose of RAIT, and negatively associated with an increasing age. The cumulative dose of RAIT was associated with mortality in a linear dosedependent fashion, with a downward trend being moderate (but more accurate) in the dose of 300 to $1,000 \mathrm{mCi}$. The increasing patient age was associated with mortality in a non-linear fashion, with the optimal threshold being 40 years. With advancing age, the risk of death increases rapidly in patients aged 40 years and younger, but slowly in patients over 40 years.

\section{DATA AVAILABILITY}

The datasets generated and/or analyzed during the current study are not publicly available due to data security but are available from the corresponding author on reasonable request.

\section{ETHICS STATEMENT}

This study was carried out in accordance with the Declaration of Helsinki and was approved by the Independent Ethics Committee of Cancer Hospital, Chinese Academy of Medical Sciences. Waiver of informed consent was applied to this study because it is a retrospective analysis of existing data.

\section{AUTHOR CONTRIBUTIONS}

RZ and Y-XL: conception and design and critical revision of the article for important intellectual content. JY: analysis and interpretation of the data and drafting of the article. JY, ML, YJ, LL, JG, and SC: collection of provisions of data. All authors: final approval of the article.

\section{FUNDING}

This study was funded by the National Key Projects of Research and Development of China (2016YFC0904600), CAMS Innovation Fund for Medical Sciences (CIFMS) (2016-I2M-1001, 2017-I2M-3-005), and the Beijing Hope Run Special Fund of Cancer Foundation of China (LC 2017A03).

\section{ACKNOWLEDGMENTS}

We thank Changfa Xia for assistance in statistical analyses.

\section{SUPPLEMENTARY MATERIAL}

The Supplementary Material for this article can be found online at: https://www.frontiersin.org/articles/10.3389/fonc. 2019.00558/full\#supplementary-material 


\section{REFERENCES}

1. Wiltshire JJ, Drake TM, Uttley L, Balasubramanian SP. Systematic review of trends in the incidence rates of thyroid cancer. Thyroid. (2016) 11:1541-52. doi: 10.1089/thy.2016.0100

2. Yang L, Zheng R, Wang N, Zhang S, Chen W. Analysis of incidence and mortality of thyroid cancer in China, 2010. Chin J Prev Med. (2014) 8:663-8. doi: 10.3760/cma.j.issn.0253-9624.2014.08.003

3. Zheng RS, Sun KX, Zhang SW, Zeng HM, Zou XN, Chen R, et al. Report of cancer epidemiology in China, 2015. Chin J Oncol. (2019) 1:19-28. doi: 10.3760/cma.j.issn.0253-3766.2019.01.005

4. Zeng $\mathrm{H}$, Chen W, Zheng R, Zhang S, Ji JS, Zou X, et al. Changing cancer survival in China during 2003-15: a pooled analysis of 17 population-based cancer registries. Lancet Glob Health. (2018) 5:e555-67. doi: 10.1016/s2214-109x(18)30127-x

5. Leite AKN, Cavalheiro BG, Kulcsar MA, Hoff AO, Brandao LG, Cernea CR, et al. Deaths related to differentiated thyroid cancer: a rare but real event. Arch Endocrinol Metab. (2017) 3:222-7. doi: 10.1590/2359-3997000000261

6. O’Neill CJ, Oucharek J, Learoyd D, Sidhu SB. Standard and emerging therapies for metastatic differentiated thyroid cancer. Oncologist. (2010) 2:146-56. doi: 10.1634/theoncologist.2009-0190

7. Song HJ, Qiu ZL, Shen CT, Wei WJ, Luo QY. Pulmonary metastases in differentiated thyroid cancer: efficacy of radioiodine therapy and prognostic factors. Eur J Endocrinol. (2015) 3:399-408. doi: 10.1530/eje-15-0296

8. Pacini F, Castagna MG, Brilli L, Pentheroudakis G. Thyroid cancer: ESMO Clinical Practice Guidelines for diagnosis, treatment and follow-up. Ann Oncol. (2010) 23:v214-9. doi: 10.1093/annonc/mdq190

9. Haugen BR, Alexander EK, Bible KC, Doherty GM, Mandel SJ, Nikiforov YE, et al. 2015 American thyroid association management guidelines for adult patients with thyroid nodules and differentiated thyroid cancer: the American thyroid association guidelines task force on thyroid nodules and differentiated thyroid cancer. Thyroid. (2016) 1:1-133. doi: 10.1089/thy. 2015.0020

10. Ronga G, Filesi M, Montesano T, Di Nicola AD, Pace C, Travascio L, et al. Lung metastases from differentiated thyroid carcinoma. A 40 years' experience. Q J Nucl Med Mol Imaging. (2004) 1:12-9.

11. Haugen BR, Sherman SI. Evolving approaches to patients with advanced differentiated thyroid cancer. Endocr Rev. (2013) 3:439-55. doi: 10.1210/er.2012-1038

12. Martins-Filho R, Ward LS, Amorim BJ, Santos AO, Lima MC, Ramos CD, et al. Cumulative doses of radioiodine in the treatment of differentiated thyroid carcinoma: knowing when to stop. Arq Bras Endocrinol Metabol. (2010) 9:807-12. doi: 10.1590/S0004-27302010000900006

13. Pitoia F, Bueno F, Cross G. Long-term survival and low effective cumulative radioiodine doses to achieve remission in patients with 131Iodine-avid lung metastasis from differentiated thyroid cancer. Clin Nucl Med. (2014) 9:784-90. doi: 10.1097/rlu.0000000000000507

14. Nakanishi K, Kikumori T, Miyajima N, Takano Y, Noda S, Takeuchi D, et al. Impact of patient age and histological type on radioactive iodine avidity of recurrent lesions of differentiated thyroid carcinoma. Clin Nucl Med. (2018) 7:482-5. doi: 10.1097/rlu.0000000000002078

15. Pawelczak M, David R, Franklin B, Kessler M, Lam L, Shah B. Outcomes of children and adolescents with well-differentiated thyroid carcinoma and pulmonary metastases following (1)(3)(1)I treatment: a systematic review. Thyroid. (2010) 10:1095-101. doi: 10.1089/thy.2009.0446

16. Wang R, Zhang Y, Tan J, Zhang G, Zhang R, Zheng W, et al. Analysis of radioiodine therapy and prognostic factors of differentiated thyroid cancer patients with pulmonary metastasis: an 8-year retrospective study. Medicine (Baltimore). (2017) 19:e6809. doi: 10.1097/md.000000000000 6809

17. Isoda $\mathrm{T}, \mathrm{BaBa} \mathrm{S}$, Maruoka $\mathrm{Y}$, Kitamura Y, Tahara K, Sasaki M, et al. Impact of patient age on the iodine/FDG "flip-flop" phenomenon in lung metastasis from thyroid cancer. Ann Nucl Med. (2016) 8:518-24. doi: 10.1007/s12149-016-1104-9

18. Mazurat A, Torroni A, Hendrickson-Rebizant J, Benning H, Nason RW, Pathak KA. The age factor in survival of a population cohort of well-differentiated thyroid cancer. Endocr Connect. (2013) 3:154-60. doi: 10.1530/ec-13-0056
19. Tran B, Roshan D, Abraham E, Wang L, Garibotto N, Wykes J, et al. An analysis of the American joint committee on cancer 8th edition T staging system for papillary thyroid carcinoma. J Clin Endocrinol Metab. (2018) 6:2199-206. doi: 10.1210/jc.2017-02551

20. van Velsen EFS, Stegenga M, van Kemenade FJ, Kam B, van Ginhoven TM, Visser WE, et al. Comparing the prognostic value of the eighth edition of the AJCC/TNM staging system between papillary and follicular thyroid cancer. Thyroid. (2018) 28:976-81. doi: 10.1089/thy.2018.0066

21. Verburg FA, Mader U, Luster M, Reiners C. The effects of the Union for International Cancer Control/American Joint Committee on Cancer Tumour, node, metastasis system version 8 on staging of differentiated thyroid cancer: a comparison to version 7. Clin Endocrinol (Oxf). (2018) 6:950-6. doi: $10.1111 /$ cen. 13597

22. Ganly I, Nixon IJ, Wang LY, Palmer FL, Migliacci JC, Aniss A, et al. Survival from differentiated thyroid cancer: what has age got to do with it? Thyroid. (2015) 10:1106-14. doi: 10.1089/thy.2015.0104

23. Adam MA, Thomas S, Hyslop T, Scheri RP, Roman SA, Sosa JA. Exploring the relationship between patient age and cancer-specific survival in papillary thyroid cancer: rethinking current staging systems. J Clin Oncol. (2016) 36:4415-20. doi: 10.1200/jco.2016.68.9372

24. Chen P, Feng HJ, Ouyang W, Wu JQ, Wang J, Sun YG, et al. Risk factors for nonremission and progression-free survival after I-131 therapy in patients with lung metastasis from differentiated thyroid cancer: a single-institute, retrospective analysis in southern China. Endocr Pract. (2016) 9:1048-56. doi: 10.4158/ep151139.or

25. Chopra S, Garg A, Ballal S, Bal CS. Lung metastases from differentiated thyroid carcinoma: prognostic factors related to remission and disease-free survival. Clin Endocrinol (Oxf). (2015) 3:445-52. doi: 10.1111/cen.12558

26. Hindie E, Melliere D, Lange F, Hallaj I, de Labriolle-Vaylet C, Jeanguillaume C, et al. Functioning pulmonary metastases of thyroid cancer: does radioiodine influence the prognosis? Eur J Nucl Med Mol Imaging. (2003) 7:974-81. doi: 10.1007/s00259-003-1174-5

27. Jeong JH, Kong EJ, Jeong SY, Lee SW, Cho IH, Ah Chun K, et al. Clinical outcomes of low-dose and high-dose post-operative radioiodine therapy in patients with intermediate-risk differentiated thyroid cancer. Nucl Med Commun. (2017) 3:228-33. doi: 10.1097/mnm.0000000000000636

28. Samuel AM, Rajashekharrao B, Shah DH. Pulmonary metastases in children and adolescents with well-differentiated thyroid cancer. J Nucl Med. (1998) 9:1531-6.

29. Wartofsky L, Van Nostrand D. Radioiodine treatment of well-differentiated thyroid cancer. Endocrine. (2012) 3:506-13. doi: 10.1007/s12020-0129729-5

30. Chiesa C, Castellani MR, Vellani C, Orunesu E, Negri A, Azzeroni R, et al. Individualized dosimetry in the management of metastatic differentiated thyroid cancer. Q J Nucl Med Mol Imaging. (2009) 5:546-61.

31. Medvedec M. Thyroid stunning in vivo and in vitro. Nucl Med Commun. (2005) 8:731-5. doi: 10.1097/01.mnm.0000169380. 92270.73

32. Handkiewicz-Junak D, Wloch J, Roskosz J, Krajewska J, Kropinska A, Pomorski L, et al. Total thyroidectomy and adjuvant radioiodine treatment independently decrease locoregional recurrence risk in childhood and adolescent differentiated thyroid cancer. J Nucl Med. (2007) 6:879-88. doi: 10.2967/jnumed.106.035535

33. Capdevila J, Galofre JC, Grande E, Zafon Llopis C, Ramon YCAT, Navarro Gonzalez E, et al. Consensus on the management of advanced radioactive iodine-refractory differentiated thyroid cancer on behalf of the Spanish Society of Endocrinology Thyroid Cancer Working Group (GTSEEN) and Spanish Rare Cancer Working Group (GETHI). Clin Transl Oncol. (2017) 3:279-87. doi: 10.1007/s12094-016-1554-5

34. Sherman SI, Jarzab B, Cabanillas ME, Licitra LF, Pacini F, Martins R, et al. A phase II trial of the multitargeted kinase inhibitor E7080 in advanced radioiodine (RAI)-refractory differentiated thyroid cancer (DTC). J Clin Oncol Off J Am Soc Clin Oncol. (2011) 15(suppl):5503.

35. Ylli D, Burman KD, Van Nostrand D, Wartofsky L. Eliminating the age cutoff in staging of differentiated thyroid cancer: the safest road? J Clin Endocrinol Metab. (2018) 5:1813-7. doi: 10.1210/jc.2017-02725

36. Qiu ZL, Shen CT, Luo QY. Clinical management and outcomes in patients with hyperfunctioning distant metastases from differentiated thyroid cancer 
after total thyroidectomy and radioactive iodine therapy. Thyroid. (2015) 2:229-37. doi: 10.1089/thy.2014.0233

37. Wu S, Wang H. Efficacy analysis of (131)I therapy and predictive value of preablation stimulated thyroglobulin for lung metastases from differentiated thyroid cancer. Ann Endocrinol (Paris). (2013) 1:40-4. doi: 10.1016/j.ando.2012.11.007

38. Zhang X, Liu DS, Luan ZS, Zhang F, Liu XH, Zhou W, et al. Efficacy of radioiodine therapy for treating 20 patients with pulmonary metastases from differentiated thyroid cancer and a meta-analysis of the current literature. Clin Transl Oncol. (2018) 7:928-35. doi: 10.1007/s12094-017-1 $792-1$

39. Orosco RK, Hussain T, Brumund KT, Oh DK, Chang DC, Bouvet M. Analysis of age and disease status as predictors of thyroid cancer-specific mortality using the surveillance, epidemiology, and end results database. Thyroid. (2015) 1:125-32. doi: 10.1089/thy.201 4.0116
40. Tang J, Kong D, Cui Q, Wang K, Zhang D, Liao X, et al. Racial disparities of differentiated thyroid carcinoma: clinical behavior, treatments, and longterm outcomes. World J Surg Oncol. (2018) 1:45. doi: 10.1186/s12957-018$1340-7$

Conflict of Interest Statement: The authors declare that the research was conducted in the absence of any commercial or financial relationships that could be construed as a potential conflict of interest.

Copyright (๑) 2019 Yang, Zheng, Liang, Jia, Lin, Geng, Chen and Li. This is an open-access article distributed under the terms of the Creative Commons Attribution License (CC BY). The use, distribution or reproduction in other forums is permitted, provided the original author(s) and the copyright owner(s) are credited and that the original publication in this journal is cited, in accordance with accepted academic practice. No use, distribution or reproduction is permitted which does not comply with these terms. 